\title{
Phytoremediation of Light Non-Aqueous Phase Liquids
}

\author{
Sunday Oniosun' ${ }^{1}$, Michael Harbottle ${ }^{1}$, Snehasis Tripathy ${ }^{1}$, Peter Cleall ${ }^{1}$.
}

\begin{abstract}
Non-aqueous phase liquids (NAPLs) are persistent sources of contamination in the ground, providing a long-term supply of dissolved phase contamination and taking significant periods to dissipate naturally. Light NAPLs (LNAPLs) take the form of a separate phase within the ground, often as individual ganglia in pore spaces within the capillary zone such that the contaminated region is diffuse and comprised of many unconnected small contaminant sources. Consequently, remedial action is challenging and success may be limited to ex-situ remediation techniques. The ability of plants to phytoremediate dissolved-phase contamination is well known, but the impact of LNAPLs on plant growth and subsequent contaminant behaviour is largely unknown. Experimental work with ryegrass (Lolium perenne) is presented, exploring the impact of the physical presence of an LNAPL (mineral oil) on plant growth, root distribution and oil removal. The presence of the oil was found to significantly impact root biomass and distribution, leading to zones of increased root growth alongside decreased shoot growth. Significant removal of the LNAPL was noted in both hydroponic conditions and planted soil.
\end{abstract}

Keywords: phytoremediation, ryegrass, mineral oil, non-aqueous phase liquids.

\subsection{Introduction}

Phytoremediation of organic contamination in soils may arise as a result of contaminant uptake into plant tissues [1,2], phytovolatilization [3] or biodegradation of contaminants by rhizosphere-associated microorganisms [4]. Success has been reported in the remediation of a range of organic contaminant families - for example fuel oils [5], chlorophenols [1] and polyaromatic hydrocarbons [6]. However, phytoremediation studies typically do not report the form of the contamination, i.e. is it present as a non-aqueous phase liquid (NAPL) or in the dissolved phase? It is to be expected that NAPLs (particularly light, or LNAPLs) might impact on plant growth

\footnotetext{
${ }^{1}$ M. Harbottle $(\bowtie)$

School of Engineering, Cardiff University, Cardiff, United Kingdom e-mail: harbottlem@cardiff.ac.uk
} 
and survivability, not just through their toxicity but also through their physical presence in the pore space and their ability to act as a barrier to moisture and solutes.

The impact of NAPLs on plants, and the potential for plants to tackle NAPL contamination, is unknown. Zhang et al [7] explored uptake of trichloroethylene, introduced in NAPL form, by alfalfa although the impact of NAPL on the plant is unknown and impacts on the contaminant may have been limited to the dissolved phase. Previous studies on organic contaminants may have involved sufficient concentrations that NAPLs were present but this is not specifically reported. NAPLs are challenging to remediate [8] but if phytoremediation were capable of treating this form of contamination, then this may potentially be a valuable mechanism of remediation for LNAPLs in soils accessible by plant root systems. This work has explored the impact of the presence of LNAPL on plant growth, alongside determining the degree of oil removal due to the presence of the plant. Two studies are presented-hydroponic microcosms mimicked the soil pore structure to explore the impact of NAPL on individual plant root systems, whilst larger scale mesocosms have explored phytoremediation and impact of oil on plants in artificial soil systems.

\subsection{Impact of NAPLs on Root Distribution and Growth, and Oil Loss, at the Root Scale}

The response of grass plants to oil in a hydroponic system has been explored to understand the impacts on root growth and distribution, as well as oil removal.

\subsubsection{Experimental Design and Methodology}

Perennial ryegrass (Lolium perenne) is capable of phytoremediation of organic contaminants [9] and grew reliably in the experimental conditions. Quarter strength Hoagland's solution (2.5 g/L Hoagland's No.2 Basal Salt Mixture (Sigma-Aldrich, UK) in deionized water) was used as a hydroponic solution. Mineral oil (Fisher BioReagents) was chosen as the model LNAPL. Its low toxicity, volatility and aqueous solubility minimised the impact of NAPL toxicity on plant growth (ensuring the main impacts are due to physical presence), and ensured that any contaminant loss was down to biodegradation rather than solubilisation or volatilisation. The oil was coloured with $50 \mathrm{mg} / \mathrm{L}$ Oil Red O (Sigma-Aldrich) to enhance visibility.

Plant growth microcosms (Figure 1) were 3D-printed from polylactic acid. Each microcosm comprised four vertical columns to represent soil pore channels, above which a ' $v$ ' shaped housing held a plant seed whilst allowing roots to progress downwards through the open apex of the housing. The front face of the microcosm was covered with a thin sheet of acetate film for visual monitoring, apart from over small area at the base. The construction materials only minimally absorbed the oil. 


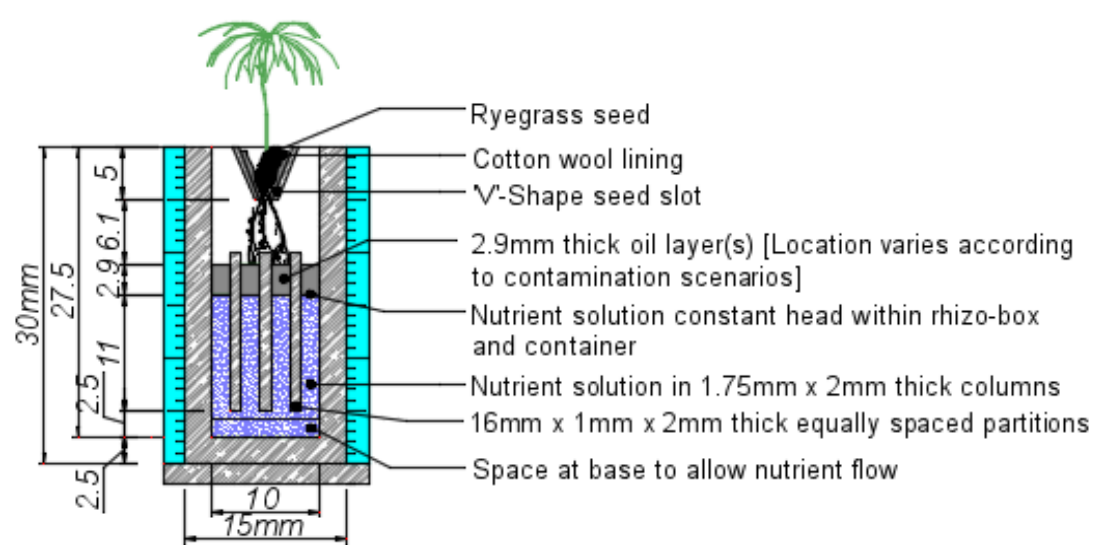

Figure 1. Plant growth microcosm schematic diagram.

Experiments explored root growth in two scenarios with and without mineral oil. Each scenario had five replicates, with the ten microcosms secured to the base of a plastic reservoir filled to a depth of $20 \mathrm{~mm}$ with the Hoagland's solution - the opening at the base of the acetate film allowed this level to be maintained within the microcosm. This level was maintained using a Mariotte bottle system, whilst the reservoir fluid was pumped through an ultraviolet steriliser at approximately one volume per twelve hours to control algal growth. In the scenario with oil, each column had $10 \mu \mathrm{l}$ mineral oil placed on the surface of the fluid by syringe. A single seed of ryegrass was placed in the seed housing and secured with a small amount of cotton wool soaked in Hoagland's solution. The planted microcosms were subjected to an artificial daylight regime from $58 \mathrm{~W}$ cool white daylight spectrum fluorescent tubes, located $1.5 \mathrm{~m}$ above the microcosms, for 16 hours per day over four weeks.

Following this period, oil levels were observed visually and scored (all oil remaining $=1$, partial oil $=0.5$, no oil $=0$ ) as the oil layer was not sufficiently thick to allow accurate measurement. Similarly, root growth in each of the four columns (numbered 1-4 from left to right) was observed and scored (established root growth $=1$, partial root growth $=0.5$, no root $=0$ ). Subsequently, the microcosms were dismantled then roots and shoots separated and total weight and length recorded.

\subsubsection{Results and Discussion}

Root growth was observed to be non-uniform, with significant growth in the central columns ( 2 and 3 , directly below the seed housing) when no oil was present whilst that for the peripheral columns ( 1 and 4 ) was much smaller, with only one microcosm in each showing partial growth (Figure 2a). With oil present, the roots were better distributed across all columns, although there was still greater presence in the centre. This corresponds to typically greater oil loss in columns 2 and 3 with lower 
oil removal in the peripheral columns (1 and 4). This suggests that whilst the presence of a root in a column corresponds to oil removal, even roots in adjacent columns appear to cause phytoremediation, albeit more slowly than when a plant root was present. Oil loss without plants was found to be minimal (results not presented), inferring that the process of oil loss is biological.

Root and shoot masses and lengths are shown in Figure $2 b$. The presence of oil has a detrimental effect on both shoot mass and length. Root lengths are, on average, comparable but there is larger root mass with oil present. This may be explained by observations of thicker roots in the presence of oil, suggesting that this causes the plant to put increased effort into root development at the expense of shoot growth.

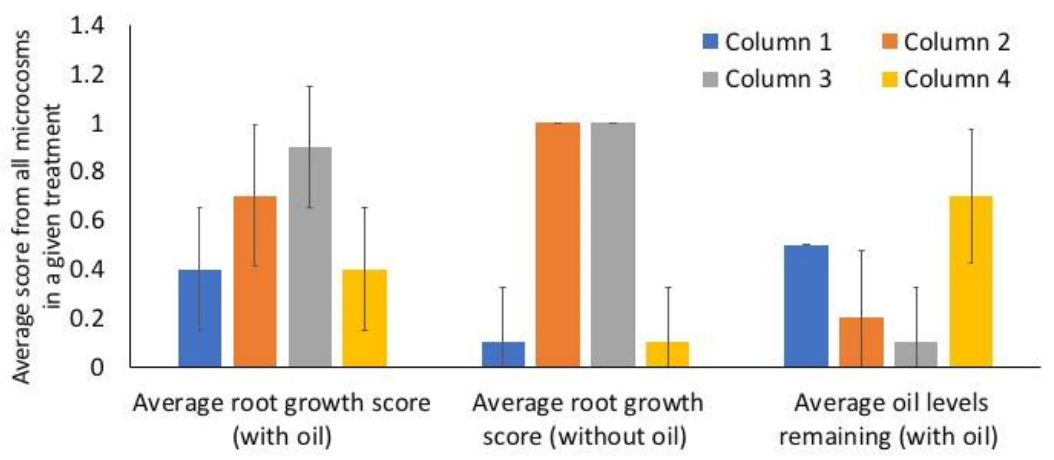

(a)

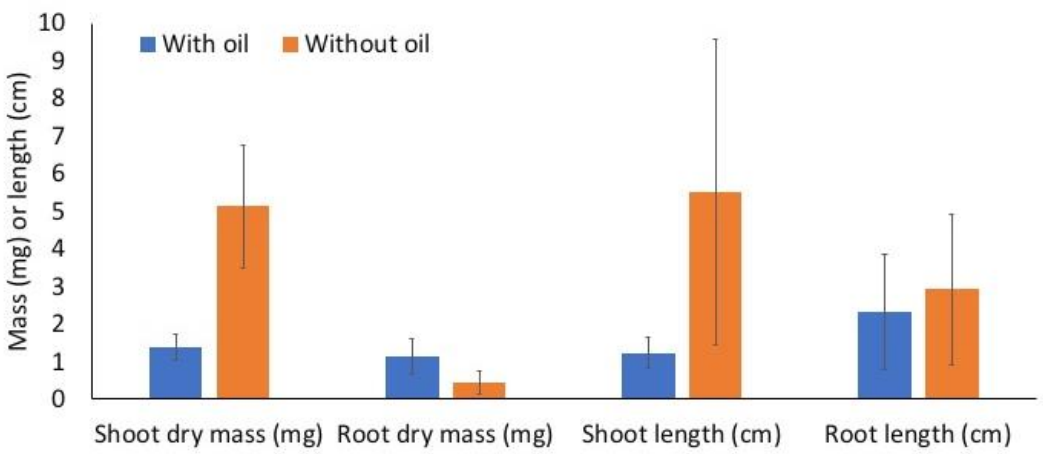

(b)

Figure 2. (a) Scoring of root growth and oil loss in individual columns. (b) Final shoot and root masses and lengths.

\subsection{Impact of NAPLs on Plant Growth in Soil at the Meso-Scale}

The behaviour observed in microcosms was explored at a larger scale in soil mesocosms to determine behaviour of a planted soil and effect on NAPL contamination. 


\subsubsection{Experimental Design and Methodology}

A sandy loam was prepared by mixing sand $(70 \%$ by mass Leighton Buzzard Garside medium sand, 0.18-2.00 mm), silt (20\% by mass Leighton Buzzard Garside fine sand, $<0.06 \mathrm{~mm}$ ) and clay (Speswhite kaolin, Imerys) in the laboratory. The components were thoroughly mixed using an electric mixer and the resulting soil characterized following British Standard methods [10] to determine liquid (13.0\%) and plastic limits (10.6\%), hydraulic conductivity $\left(1.5 \times 10^{-4} \mathrm{~m} / \mathrm{s}\right)$ and $\mathrm{pH}(7.9)$.

Mineral oil (with Oil Red O) was again used as a model LNAPL, perennial ryegrass (Lolium perenne) was used as the model plant and quarter-strength Hoagland's solution was employed for nutrient supply. Experiments were conducted in acrylic plant growth chambers with internal dimensions $25 \times 250 \times 350 \mathrm{~mm}$ (Figure 3). A central partition separated the chamber into two segments, although in these experiments conditions on either side were identical. A $40 \mathrm{~mm}$ thick free-draining gravel layer (4-12 $\mathrm{mm}$ ) was placed at the bottom of the chamber and covered with a layer of Whatman filter paper to prevent mixing with the sandy loam. Water levels within the chamber were controlled using an external plastic tube connected by flexible tubing to a port located in the gravel layer. The water level in this was maintained at a constant height, maintaining the 'groundwater' level within the soil.

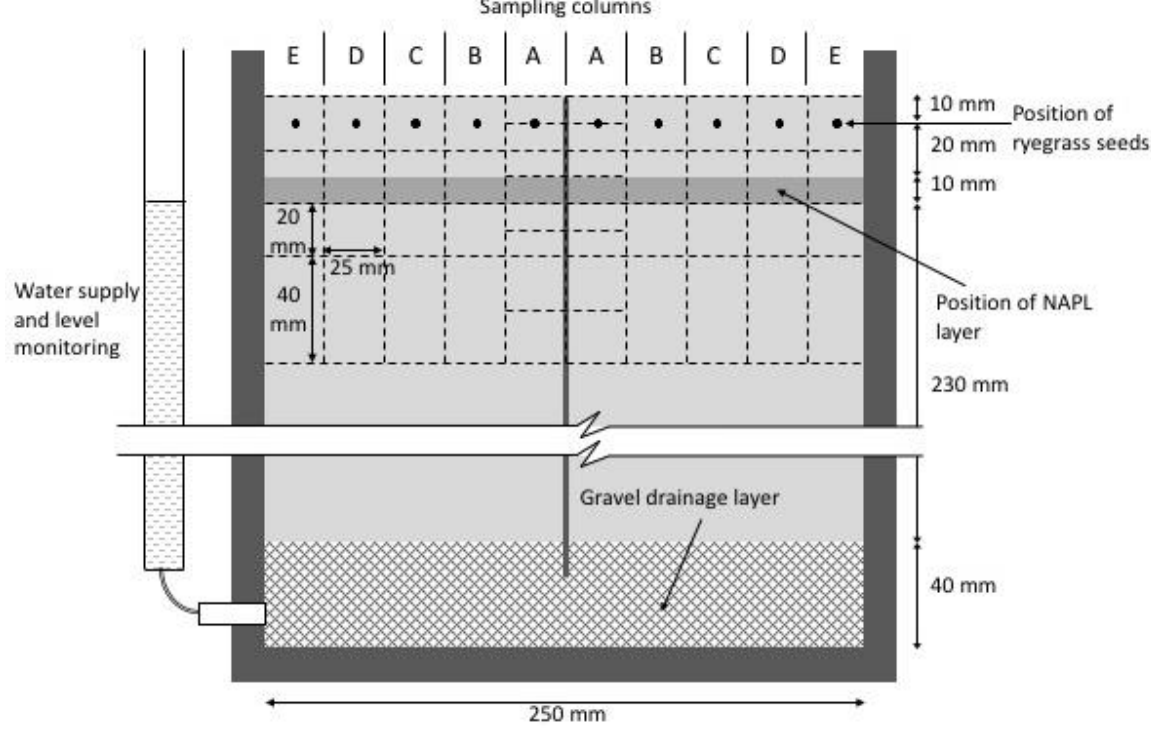

Figure 3. Plant growth chamber diagram. Dashed lines represent sample cutting locations.

Sandy loam was placed in two plant growth chambers by firstly wet-packing a $230 \mathrm{~mm}$ thick layer with the moisture content at the plastic limit to a density of 1.75 $\mathrm{g} / \mathrm{cm}^{3}$ before shaking on a mechanical shaker for 2 seconds. This was then saturated via the plastic tube (i.e. from the base) with quarter-strength Hoagland's solution. 
Next, a $10 \mathrm{~mm}$ thick layer of soil was placed on top, then lightly compacted to the target density. In one plant growth chamber this had a moisture content equivalent to the plastic limit as before. In the other, the soil was mixed with mineral oil to an oil content equivalent to the plastic limit. A further $20 \mathrm{~mm}$ of uncontaminated moistened soil was placed above this, with ryegrass seeds spread on top to a density of $50 \mathrm{~g} / \mathrm{m}^{2}$. This was then covered by a further $10 \mathrm{~mm}$ layer of moistened soil.

Over the ten weeks of the experiment, quarter-strength Hoaglands solution was added via the supply tube every day to maintain the water table at the same position. The plant growth chambers were wrapped with aluminum foil to protect roots from light and subjected to lighting and environmental conditions as for the microcosm experiments. Following the experiment the shoots were cut for each of the ten columns (A-E, left and right sides) and stored before freezing of the plant growth chambers at $-20{ }^{\circ} \mathrm{C}$. After removal of the front cover of the chamber the frozen soil was cut into the sections (see Figure 3 ) with a Starrett band saw blade ( $2 \mathrm{~mm}$ tooth pitch). Individual samples were frozen in resealable bags prior to analysis.

Frozen soil samples were mixed with distilled water (approximately 1 to 4 by volume) in petri dishes. The mineral oil content of each sample was determined using hydrophobic oil-absorbent matting (MAT440, New Pig Ltd) [11] - a piece of mat was placed in the dish, covered and the sample shaken on an orbital shaker for 24 hours. The difference in weight of the matting over this period was reported as the mass of oil present in the sample. In preliminary tests the matting was found to recover 80 and $99 \%$ of the oil present in soil samples with 0.01 and $20 \%$ oil by mass using this method. Root material was collected by placing the soil/water mixture in a sieve (200 $\mu \mathrm{m}$ aperture) and submerging this in water such that the floating root matter could be collected and dried before weighing.

\subsubsection{Results and Discussion}

The biomass present in the oil-free mesocosm after 10 weeks is shown in Figure 4a. Data are presented for both the left and right side of the central partition, in columns A-E (A closest to partition). Root matter decreases with depth, which is particularly clearly illustrated with the A samples. The discrepancy between the A samples and the B-E samples is because the former were taken every centimetre rather than every two centimetres as the remainder were. From the plot of root mass with depth, as well as the sub-plots of total root and shoot mass for each column, it is clear that there is very uniform growth across all sampling columns.

Figure $4 \mathrm{~b}$ is presented similarly, although the grey bar shows the location of the NAPL and oil loss is presented alongside the plot of total root biomass. Between 50 and $70 \%$ of the oil was removed, demonstrating substantial phytoremediation of non-aqueous phase contamination. Once more, there was generally uniform growth across the mesocosm, both above and below the soil surface. As with the microcosm experiments, there is a decrease in the overall mass of shoots in the presence of oil, 
but there is a significant increase in the root mass, particularly in the uppermost two $\mathrm{cm}$ and just below the NAPL. The latter effect was observed at all depths where roots were found, and root mass was also found at slightly greater depth. The increased root growth may be a response of the plants to environmental stress, increasing the spread of roots in an effort to find an uncontaminated route to nutrient supply, as was observed in the microcosms. The mass increase was partially caused by an observed thickening of roots (again also observed in microcosms). Changes in root architecture (length, thickness and branching) are commonly observed as a result of abiotic stresses such as drought, salinity or metal contamination [12] although the actual impact is highly species dependent.

(a)
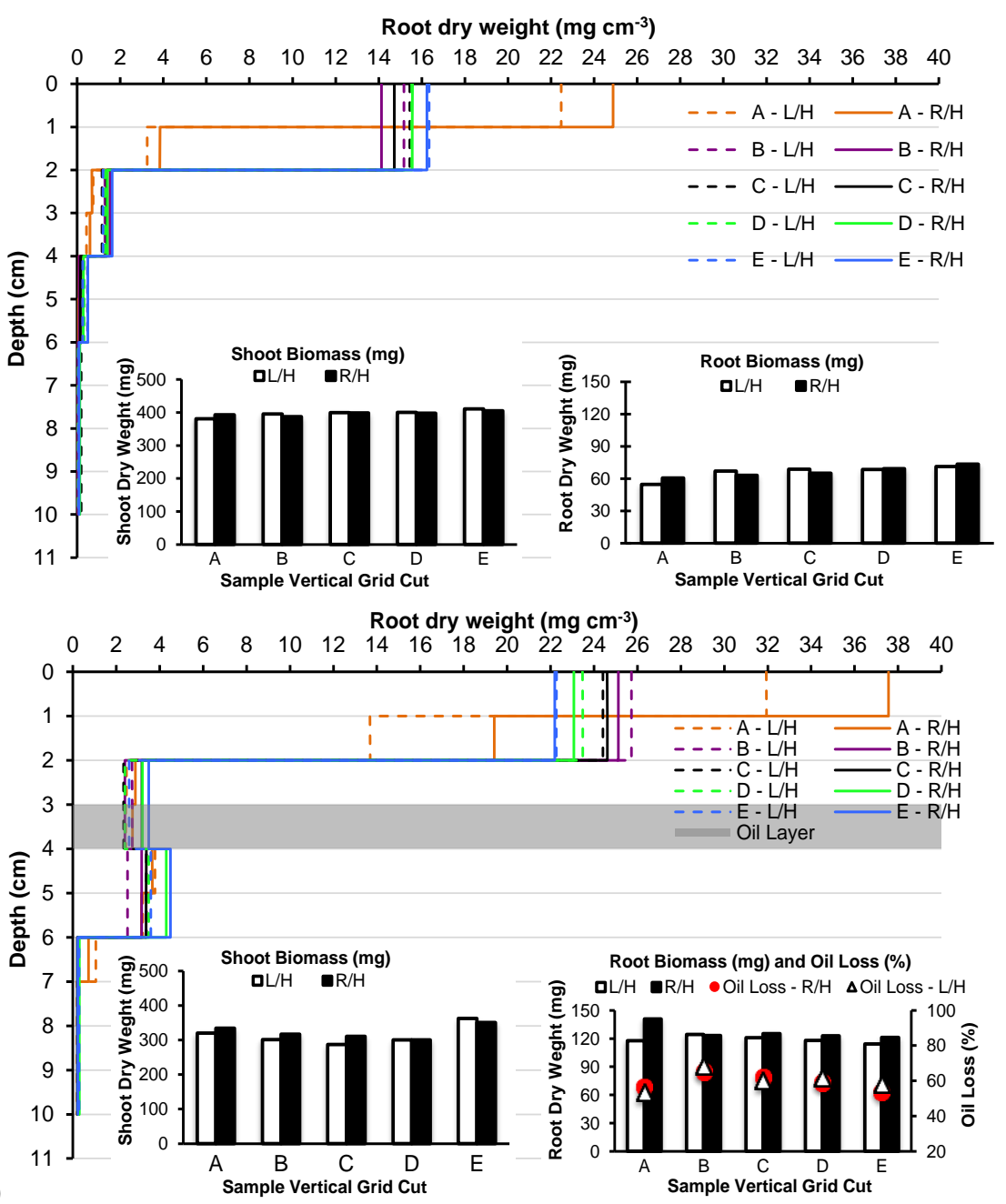

Figure 4. Root and shoot mass distribution (a) without oil, and (b) with oil. 


\subsection{Conclusions}

Two experiments at different scales have demonstrated consistent effects of the presence of an LNAPL (mineral oil) on the development of perennial ryegrass, and subsequently the removal of the oil by the plant. Both hydroponically grown single plants and multiple plant systems grown in artificial sandy loam soil demonstrate an increase in root biomass (both through increased root numbers and distribution, as well as root thickening) and a decrease in shoot biomass as a result of exposure to the LNAPL. The effect on the root system architecture is consistent with effects observed in other species due to various environmental stresses. Plant growth and metabolism resulted in removal of significant quantities of oil from the system, indicating that perennial ryegrass, and potentially other plant species too, is capable of the phytoremediation of non-aqueous phase liquids.

\subsection{References}

1. Ucisik AS, Trapp S (2008) Uptake, removal, accumulation and phytotoxicity of 4-chlorophenol in willow trees. Archives of Environmental Contamination and Toxicology, 54: 619-627

2. Zhu L, Zhang M (2008) Effect of rhamnolipids on the uptake of PAHs by ryegrass. Environmental Pollution 156:46-52

3. Huang XD, El-Alawi Y, Gurska J et al (2005) A multi-process phytoremediation system for decontamination of persistent total petroleum hydrocarbons (TPHs) from soils. Microchemical Journal 81:139-147

4. Gurska J, Wang WX, Gerhardt KE et al (2009) Three year field test of a plant growth promoting rhizobacteria enhanced phytoremediation system at a land farm for treatment of hydrocarbon waste. Environmental Science and Technology 43:4472-4479

5. Afzal M, Yousaf S, Reichenauer TG et al (2011) Soil type affects plant colonization, activity and catabolic gene expression of inoculated bacterial strains during phytoremediation of diesel. Journal of Hazardous Materials 186:1568-1575

6. Rezek J, Wiesche CID, Mackova M et al (2008) The effect of ryegrass (Lolium perenne) on decrease of PAH content in long term contaminated soil. Chemosphere 70:1603-1608

7. Zhang Q, Goplen B, Vanderhoof S et al (1996) Fate and effect of trichloroethylene as nonaqueous phase liquid in chambers with alfalfa. Proceedings of $26^{\text {th }}$ Annual Biochemical Engineering Symposium, Kansas State University, US.

8. Soga K, Page JWE, Illangasekare TH (2004) A review of NAPL source zone remediation efficiency and the mass flux approach. Journal of Hazardous Materials 110:13-27

9. Kechavarzi C, Pettersson K, Leeds-Harrison P et al (2007) Root establishment of perennial ryegrass (L. perenne) in diesel contaminated subsurface soil layers. Environmental Pollution 145:68-74

10. British Standards Institution (1990) Methods of test for soils for civil engineering purposes. BS 1377:1990.

11. Al-Ansary M, Al-Tabbaa A (2007) Stabilisation/solidification of synthetic petroleum drill cuttings. Journal of Hazardous Materials 141:410-421.

12. Franco JA, Banon S, Vicente MJ et al (2011) Root development in horticultural plants grown under abiotic stress conditions - a review. Journal of Horticultural Science and Biotechnology $86: 543-556$ 